\title{
Ultrastructural Evidence Related to Presynaptic Inhibition of Primary Muscle Afferents in Clarke's Column of the Cat
}

\author{
B. Walmsley, E. Wieniawa-Narkiewicz, ${ }^{a}$ and M. J. Nicol ${ }^{1}$ \\ Neural Research Laboratory, School of Anatomy, University of New South Wales, Kensington, N.S.W., Australia, and \\ 'Experimental Neurology Unit, John Curtin School of Medical Research, Australian National University, Canberra, A.C.T., \\ Australia
}

\begin{abstract}
As part of an investigation on excitatory synaptic transmission in the mammalian CNS, we have examined ultrastructural details of the synaptic connection between primary afferent fibers and dorsal spinocerebellar tract (DSCT) neurons in Clarke's column of the cat spinal cord. Single primary muscle afferents (group la and lb) and DSCT neurons were identified and stained intracellularly with HRP. The terminations of these afferent fibers were examined in serial sections under the EM. Five of $6 \mathrm{lb}$ boutons and 1 of $14 \mathrm{la}$ boutons were contacted by small presynaptic boutons. An example was illustrated in which only 1 out of 7 boutons arising from the same la fiber and contacting the same postsynaptic DSCT neuron was contacted by a presynaptic bouton. It is likely that the presynaptic contacts are responsible for presynaptic inhibition of synaptic transmission between primary afferents and DSCT neurons. We have proposed that the observed differences in presynaptic contacts from bouton to bouton may be one of the causes of a nonuniformity in the probability of transmitter release between release sites at this connection.
\end{abstract}

In a previous light-microscopic study (Tracey and Walmsley, 1984), the anatomical details of the monosynaptic connection between primary muscle afferents and dorsal spinocerebellar tract (DSCT) neurons in Clarke's column were examined. It was found that the synaptic terminals from both group Ia and group Ib fibers varied greatly in size, from $1 \times 1 \mu \mathrm{m}$ up to "giant" boutons, $20 \times 3 \mu \mathrm{m}$. Previous electron-microscopic studies in Clarke's column indicated that these giant boutons may contain multiple transmitter release sites, although the precise origin of the boutons was unknown (Szentagothai and Albert, 1955; Rethelyi, 1970; Saito, 1974, 1979; Houchin et al., 1983). In a recent study (Walmsley et al., 1985), the ultrastructure of identified Ia afferent terminations was examined, including the connection between Ia boutons and dendrites of a DSCT neuron, both labeled intracellularly with HRP. The results of this study confirmed earlier suggestions that Ia synaptic terminals in Clarke's column contain multiple transmitter release sites.

Received Apr. 7, 1986; revised June 30, 1986; accepted July 1, 1986.

We are grateful to Dr. E. G. Jones, Dr. D. R. Curtis, Dr. R. F. W. Fyffe, and Dr. D. J. Tracey for their comments on and suggestions concerning the manuscript. Correspondence should be addressed to Dr. B. Walmsley, Neural Research Laboratory, School of Anatomy, University of N.S.W., P.O. Box 1, Kensington, N.S.W., Australia.

a Present address: Department of Anatomy, Faculty of Medicine, The University of Calgary, 3300 Hospital Drive, N.W., Calgary, Alberta T2N 4N1, Canada.

Copyright @ 1987 Society for Neuroscience 0270-6474/87/010236-08\$02.00/0
Electrophysiological experiments have shown that excitatory postsynaptic potentials (EPSPs) evoked in DSCT neurons by impulses in a single afferent fiber fluctuate in amplitude (Tracey and Walmsley, 1984). These fluctuations occur between discrete amplitudes that are separated by quantal increments. The electron-microscopic observations on Ia terminations (Walmsley et al., 1985) are consistent with the proposal that synaptic transmission between group I muscle afferents and DSCT neurons occurs with discrete all-or-nothing EPSPs correlated with the number of release sites (rather than the number of boutons). Although quantal in nature, the fluctuation pattern of singlefiber EPSPs evoked in DSCT neurons (Walmsley et al., in press), and motoneurons (Jack et al., 1981) does not obey any standard statistical distribution, such as Poisson or binomial. Such distributions would be expected only if the probability of transmitter release were identical at all release sites. Recent evidence has been obtained (Walmsley et al., in press) that the probability of transmitter release may vary considerably from release site to release site. This observation has led us in the present electron-microscopic study to examine ultrastructural differences in the terminals of both group Ia and group Ib afferent fibers in Clarke's column. The results demonstrate the existence of presynaptic contacts on some, but not all, Ia and Ib boutons. Furthermore, boutons arising from the same Ia afferent fiber and contacting the same DSCT neuron also show differences in the occurrence of presynaptic contacts. These presynaptic contacts are most likely responsible for presynaptic inhibition in the spinal cord, and they may be one of the causes of nonuniform release probability in synaptic transmission between single muscle afferents and DSCT neurons.

\section{Materials and Methods}

Experiments were performed on cats weighing $1.5-2.5 \mathrm{~kg}$. The cats were anesthetized with sodium pentobarbital $(35 \mathrm{mg} / \mathrm{kg}$, i.p.) and maintained with supplementary doses ( $5 \mathrm{mg}$, i.v.). Mean arterial pressure and endtidal $\mathrm{CO}_{2}$ were monitored.

Identification and HRP-labeling of muscle afferents and neurons. The following muscles were exposed in the left hindlimb: medial gastrocnemius, lateral gastrocnemius, soleus, and plantaris. The tendons of these muscles were separated and cut at their insertion to allow each muscle to be stretched individually. The cat was fixcd in a rigid animal frame and a laminectomy performed from L7 to L3. The exposed hindlimb muscles and spinal cord were covered with pools of mineral oil, maintained at $37^{\circ} \mathrm{C}$ by infrared heating. Bipolar stimulating electrodes were placed on the sciatic nerve and its branches to the exposed muscles.

Glass microelectrodes containing 10\% HRP (Sigma type VI) in $1 \mathrm{M}$ $\mathrm{KCl}$ were inserted into the dorsal columns near the junction of $\mathrm{L} 3$ and L4 segments of the spinal cord, about $200 \mu \mathrm{m}$ lateral to the midline. On intracellular penetration of a primary muscle afferent, its conduction time in response to electrical stimulation of the appropriate nerve was 

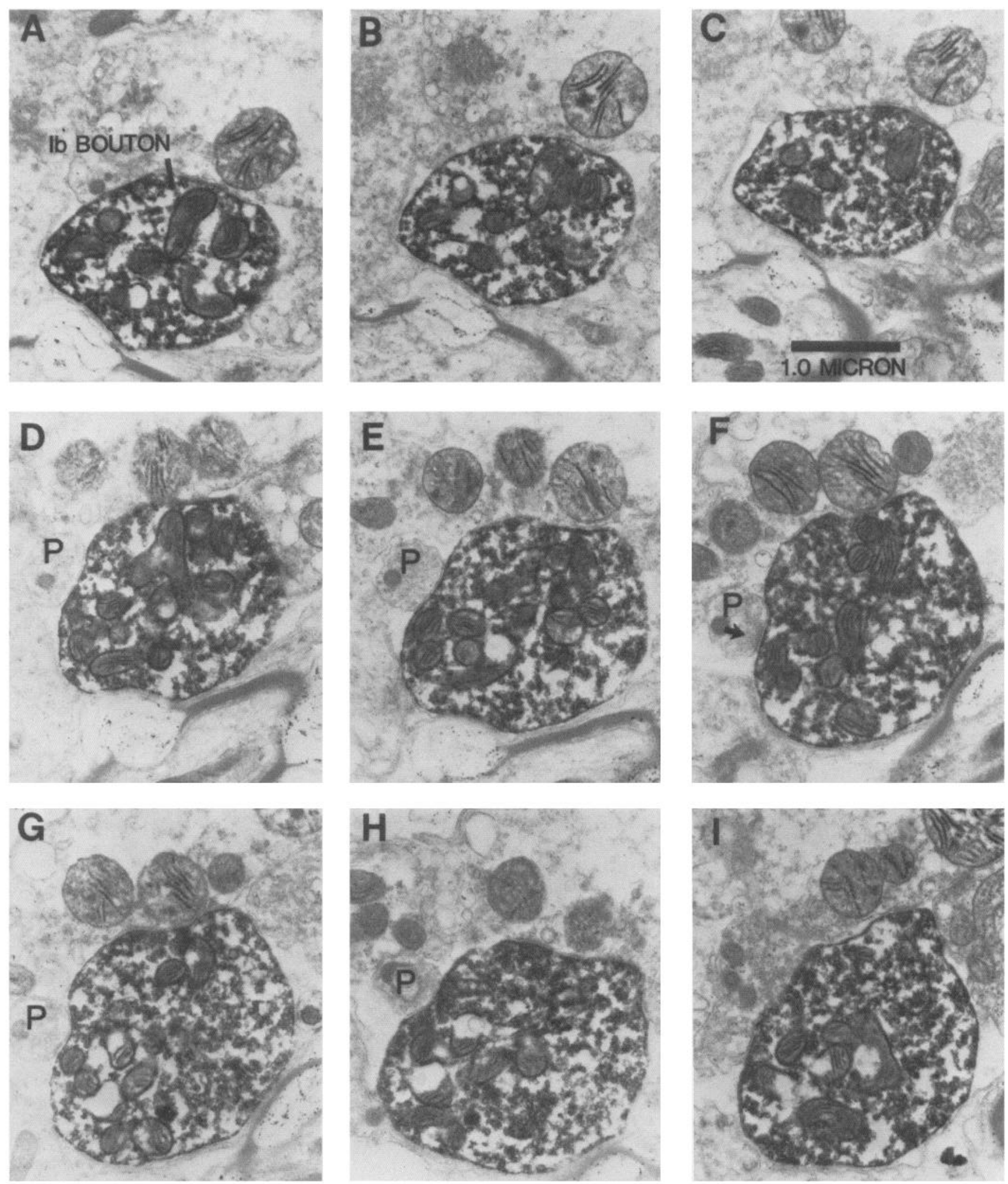

Figure 1. Serial sections through an HRP-labeled $\mathrm{Ib}$ bouton shown in sequential order $(A-I)$. A small unlabeled bouton $(P)$ contacted the $\mathrm{Ib}$ bouton (arrow in F).

recorded, allowing preliminary classification into group I or II. Fibers were classified as group Ia if they had high dynamic sensitivity to passive stretch and a silent period during active muscle contraction. Group Ib fibers had high thresholds and low dynamic sensitivity to passive stretch, and were activated during the period of a muscle twitch (Tracey and Walmsley, 1984). Once the afferent was identified, HRP was iontophoresed into the axon for up to an hour. Iontophoresis of HRP was continued only while the intracellular action potential was greater than $10 \mathrm{mV}$. 
Good results were obtained when the charge transfer was in the range $100-400 \mathrm{nA}$ min. (In any given experiment only Ia or Ib fibers, but not both, were injected with HRP.)

In the same experiments, intracellular recordings were made from DSCT neurons in Clarke's column at the level of the L3 and L4 segments of the spinal cord. Antidromic identification of the DSCT neurons was performed by stimulation of the dissected dorsolateral fasciculus of the spinal cord at the C2 level (Houchin et al., 1983; Tracey and Walmsley, 1984). The dorsal columns were removed for approximately $2 \mathrm{~cm}$ to avoid stimulation spreading to them. Electrodes filled with $10 \%$ HRP in $1 \mathrm{M} \mathrm{KCl}$ were driven into Clarke's column in the spinal cord approximately $200-300 \mu \mathrm{m}$ from the midline. Only DSCT neurons recciving monosynaptic excitation from group I musclc afferents were injected with HRP (10-30 nA for 10-40 min). The staining of both neurons and afferent fibers in the same experiment allowed subsequent identification of both pre- and postsynaptic structures under the EM.

Following a $2-5 \mathrm{hr}$ postinjection survival time, the anesthetized animal was perfused with $3 \%$ glutaraldehyde in $0.1 \mathrm{~m}$ phosphate buffer ( $\mathrm{pH}$ 7.2). The spinal cord segments $\mathrm{L} 3$ and $\mathrm{L} 4$ were removed and postfixed for about $10 \mathrm{hr}$. Parasagittal sections $(100 \mu \mathrm{m})$ of these segments were cut using a Vibratome and reacted for HRP using diaminobenzidine (DAB).

Electron microscopy. Sections immersed in glycerol were carefully examined under the light microscope. Structures filled with HRP reaction product were readily observed, even in the nondehydrated tissue. Small areas $(2 \times 1 \mathrm{~mm})$ containing stained fibers were cut from the sections and transferred to $0.1 \mathrm{~m}$ phosphate buffer $(\mathrm{pH} 7.4)$, washed, and left for $2-3 \mathrm{hr}$ at $4^{\circ} \mathrm{C}$. Subsequently, the tissue was postfixed in $2 \%$ $\mathrm{OsO}_{4}$, stained en bloc with $2 \%$ uranyl acetate, dehydrated in alcohols, and embedded in Spurr resin. Thin sections (ca. $1000 \AA$ ) were cut with a diamond knife (Diatome) on a Reichert microtome, collected on parlodion-coated slot grids, and examined in a Philips 301 EM. HRPlabeled structures were easily observed, and in the majority of cases staining was light enough not to obscure intracellular components. Sequential sections of each labeled structure were examined and relevant sections photographed. HRP-labeled boutons were examined completely in sequential sections so that the presence or absence of presynaptic structures could be confidently determined for each bouton.

\section{Results}

\section{Electron microscopy of HKP-identified Ib boutons in Clarke's column}

Six HRP-labeled Ib boutons from 2 afferent fibers were completely examined in serial sections under the EM. Figure 1 shows a sequence of sections through a Ib bouton. The HRPDAB reaction product stained this terminal darkly enough for positive identification without obscuring the major ultrastructural details. Figure 2 shows different sections through the same bouton and illustrates the main features of most Ib boutons examined. Synaptic contacts of Ib fibers showed a pronounced postsynaptic density (Fig. $2 B$ ), indicating that these synapses were of the asymmetrical type. Figure $2 C$ illustrates that this same $\mathrm{Ib}$ bouton received a contact from a small unlabeled bouton (see also Fig. 1). This was a common feature, as 5 out of $6 \mathrm{Ib}$ boutons examined were contacted by presynaptic boutons, ranging in size from 0.5 to $1.5 \mu \mathrm{m}$ in diameter. The bouton not receiving a presynaptic contact arose from the same Ib fiber that possessed terminals receiving presynaptic contacts.

Figure 3 illustrates the contacts formed between presynaptic boutons and 4 different HRP-labeled Ib boutons. One of these boutons (Fig. $3 B$ ) appeared to be contacted by 2 separate presynaptic terminals. The presynaptic terminals contained elliptical to round vesicles that accumulated close to the presynaptic membrane. Although the postsynaptic thickening was partly obscured by the HRP reaction product, these synapses appeared to be of the symmetrical type.

\section{Electron microscopy of HRP-identified Ia boutons in Clarke's column}

Fourteen boutons arising from 3 HRP-identified Ia fibers have been completely examined in serial sections under the EM. The main features of Ia boutons in Clarke's column have been described in a previous study (Walmsley et al., 1985). However, none of the boutons presented in that study was contacted by presynaptic boutons. We have now found an example of a presynaptic contact formed with a Ia bouton in Clarke's column, and this example is illustrated in Figures 4 and 5 . In this experiment, both the Ia afferent and the DSCT neuron contacted by this afferent were labeled with HRP, allowing positive identification of both pre- and postsynaptic structures. (DSCT neurons do not possess recurrent axon collaterals and therefore the possibility of confusing Ia terminals and "autapses" can be dismissed.)

Figure 4 shows 3 sequential sections through the Ia bouton (and DSCT neuron dendrite). In Figure $4 B$ the Ia bouton can be seen to make contact with a small spine arising from the DSCT neuron dendrite. In the same section, another bouton (p) appears to contact the labeled Ia bouton. This is shown in more detail in Figure 5 . Figure $5 B$ shows a schematic reconstruction of the dendrite, the Ia bouton, and the presynaptic bouton from 14 sequential sections. Figure $5 A$ is a higher-magnification electron micrograph and clearly shows the arrangement between the Ia bouton, the presynaptic bouton, and the dendritic spine arising from the DSCT neuron dendrite. In total, 7 HRP-labeled boutons arising from the same Ia fiber and contacting the dendrites of this same HRP-labeled DSCT neuron have been completely examined in serial sections under the EM. The la bouton illustrated in Figures 4 and 5 is the only 1 of the 7 to receive a presynaptic contact.

\section{Discussion}

The major findings of the present study are as follows:

1. Identified boutons of both Ia and Ib primary muscle afferents in Clarke's column are contacted by prcsynaptic boutons.

2. Not all boutons arising from a single Ia or Ib fiber are contacted by presynaptic boutons.

3. Not all boutons from a single Ia fiber that contact the same postsynaptic DSCT neuron are contacted by presynaptic boutons.

It is probable that the presynaptic contacts observed are related to presynaptic inhibition of synaptic transmission between $\mathrm{Ia}$ and $\mathrm{Ib}$ afferents and neurons in Clarke's column. Jankowska et al. $(1965,1984)$ and Curtis et al. (in press) have reported excitability changes of group I muscle afferent fibers and their terminations in Clarke's column in response to stimulation of other primary afferents. Curtis et al. (in press) proposed that primary afferent depolarization of group I muscle afferents in Clarke's column is generated at bicuculline-sensitive receptors by GABA released at axoaxonic synapses on the terminations of these afferents (see also Eccles et al., 1963; Jankowska et al., 1981; Rudomin et al., 1981; Curtis and Lodge, 1982). The present electron-microscopic study has now demonstrated the existence of such axoaxonic contacts on positively identified group Ia and $\mathrm{Ib}$ afferent boutons in Clarke's column. Such contacts have also been observed on group Ia boutons in the motor nuclei 

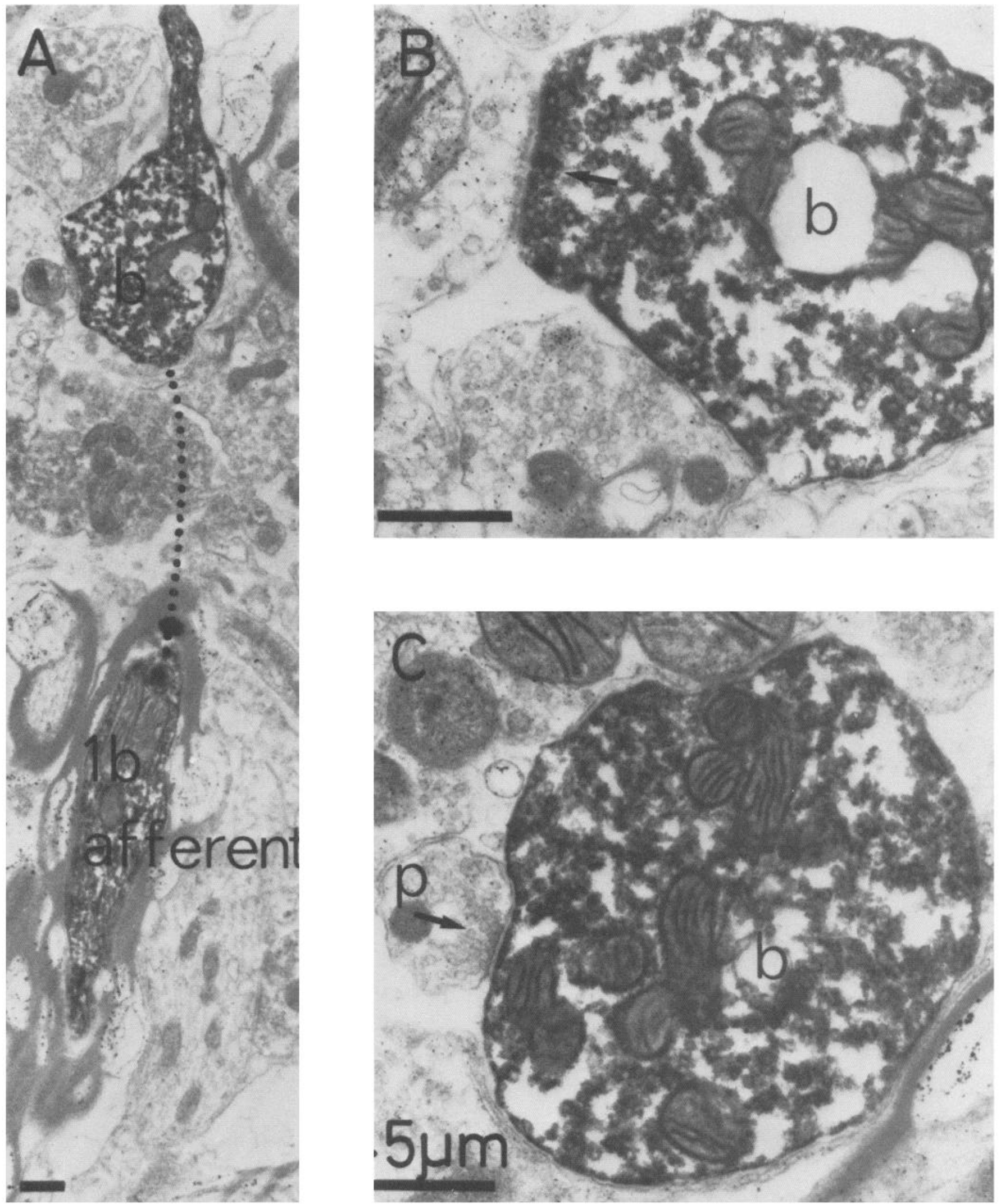

Figure 2. Photomicrographs of the same HRP-labeled bouton $(b)$ shown in Figure 1. In $A$ the afferent fiber can also be seen. The dotted line indicates the continuation of the Ib fiber that was followed in adjacent sections. $B$, Probable contact between the Ib bouton and an unlabeled postsynaptic profile. $C$, Small unlabeled bouton $(p)$ makes contact with the Ib bouton. All calibration bars, $0.5 \mu \mathrm{m}$. 

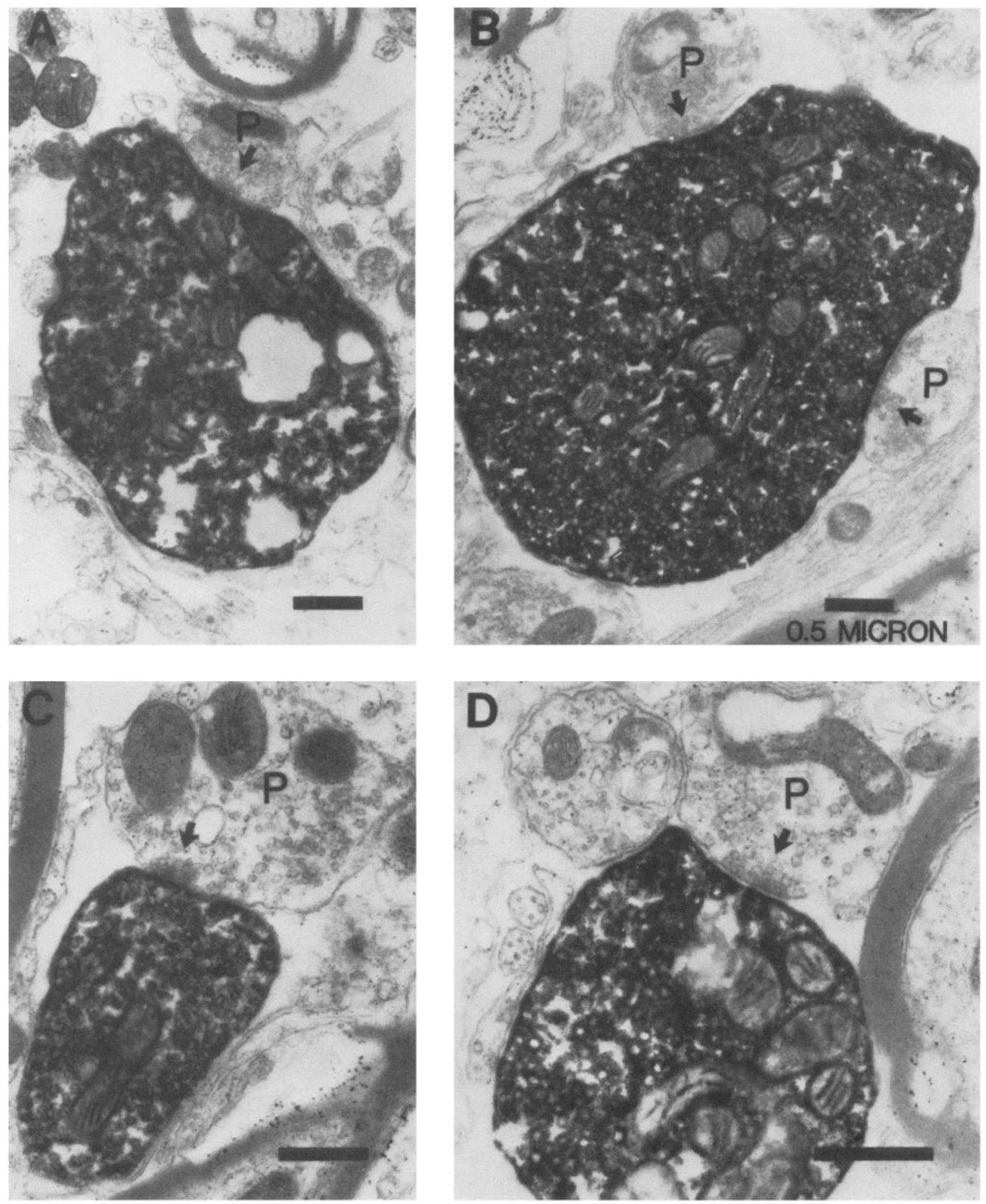

Figure 3. Presynaptic contacts between unlabeled boutons $(P)$ and 4 different HRP-labeled Ib boutons. In $B, 2$ small boutons appear to contact the same Ib bouton. All calibration bars, $0.5 \mu \mathrm{m}$.

of the cat spinal cord (Fyffe and Light, 1984), where the action of GABA has also been demonstrated (Curtis and Lodge, 1982).

Clements et al. (in press) have recently examined presynaptic inhibition of single group Ia fiber EPSPs recorded in motoneu- rons. By analyzing the fluctuations in single-fiber EPSPs, they have demonstrated that the probability of transmitter release decreases during presynaptic inhibition (see also Dudel and Kuffler, 1961). Tracey and Walmsley (1984) examined synaptic 

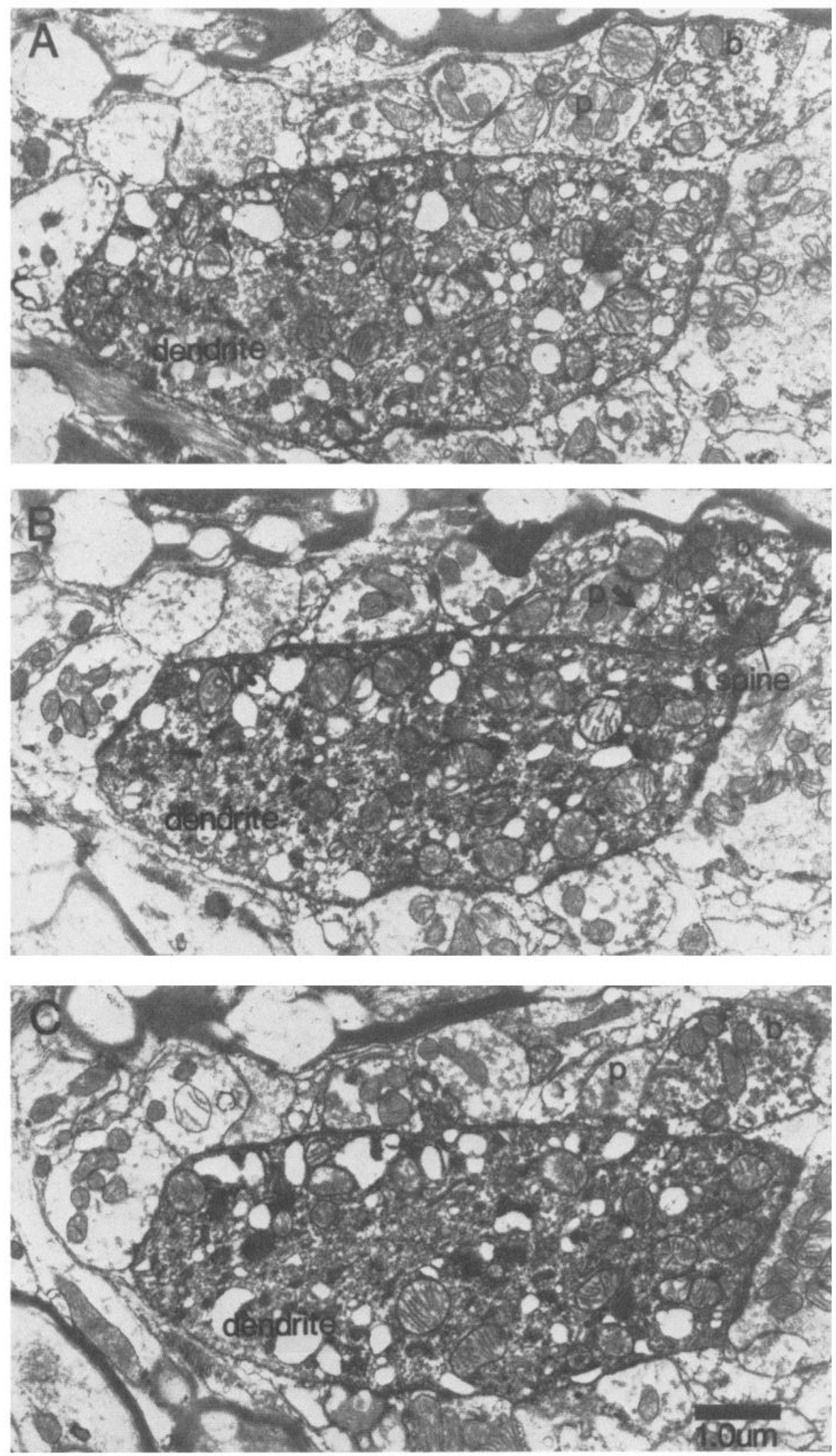

Figure 4. $A-C$, Three sequential sections through an HRP-labeled Ia bouton $(b)$ and the dendrite of an HRPlabeled DSCT neuron. The Ia bouton contacts a spine of the DSCT neuron in $B$ and, in the same section, receives a contact from an unlabeled bouton $(p)$.

transmission between single primary muscle afferents and DSCT neurons in Clarke's column. They found that single-fiber EPSPs evoked in DSCT neurons fluctuate between discrete amplitudes that are separated by quantal increments. Previous studies on synaptic transmission in the mammalian CNS have attempted to relate fluctuations in EPSP amplitude with the number of synaptic boutons (Redman and Walmsley, 1981). In contrast, Tracey and Walmsley (1984) proposed that transmission be- 
Figure 5. Details of the contact (also shown in Fig. 4) between an HRP-labeled Ia bouton and an HRP-labeled DSCT neuron dendrite. Fourteen serial sections were used in a reconstruction to obtain the schematic shown in $B$. An unlabeled bouton was found to be presynaptic to the Ia bouton (labeled in $A$ and $B$ ). The contact is indicated by the arrow in $A$.
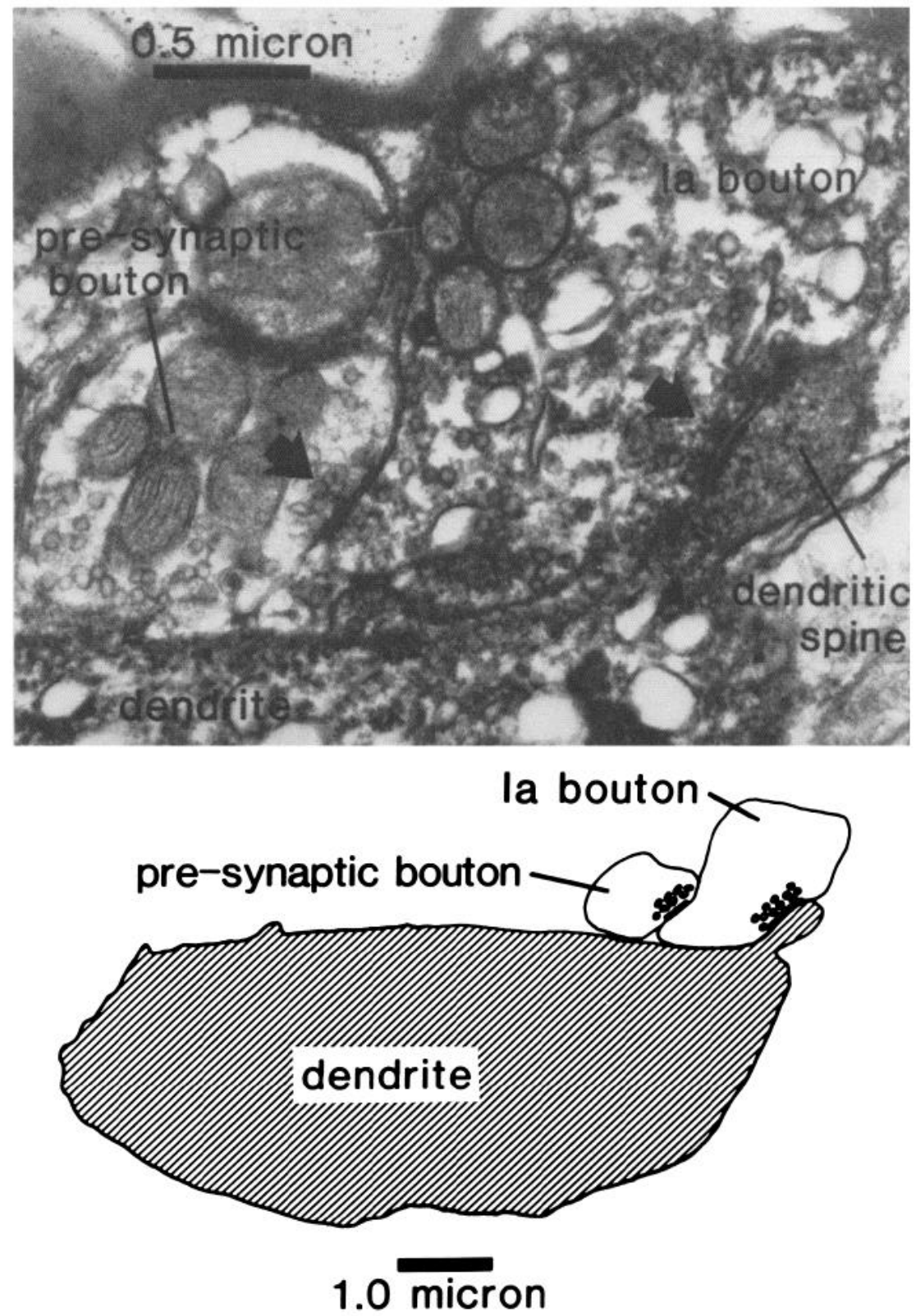

tween group I muscle afferents and DSCT neurons occurs with EPSP fluctuations related to transmitter release sites rather than boutons per se, since many boutons probably contain multiple transmitter release sites. In an EM study, Walmsley et al. (1985) demonstrated that group Ia boutons contacting DSCT neurons in Clarke's column contain multiple synaptic specializations, which probably correspond to transmitter release sites. Tracey and Walmsley (1984) concluded that the single-fiber EPSP amplitude fluctuation pattern is due to a summation of EPSPs arising from all-or-nothing transmission at individual release sites and that the probability of release may vary from release site to release site. The present electron-microscopic study has demonstrated that some boutons arising from the same single fiber may be subjected to presynaptic inhibition, and others may not. A difference in the presynaptic contacts would provide one explanation for nonuniform release probabilities. Such a scheme is illustrated in Figure 6. A presynaptic fiber is shown making contact at 2 boutons with a postsynaptic neuron. Each bouton contains 2 transmitter release sites. [The number of boutons arising from a group I muscle afferent and contacting a DSCT neuron may vary from 1 to greater than 18 (Tracey and Walmsley, 1984). In addition, the number of release sites within a single bouton may vary from 1 to greater than 9 (Walmsley et al., 1985).] Figure $6 A$ illustrates a hypothetical EPSP amplitude fluctuation pattern, assuming uniform release probabilities for all release sites. (For simplicity, the contacts and recording site in this example are considered to be somatic.) In this example, the probability has been chosen to be 0.9 (i.e., $P 1=P 2=$ $P 3=P 4=0.9$ ), and simple binomial statistics have been assumed. The EPSP amplitude may fluctuate between the 4 levels shown (plus zero), and the probabilities for each amplitude level are illustrated. Figure $6 B$ illustrates the situation in which only one of these boutons (b2) is subjected to presynaptic inhibition. The probability of transmitter release from b2 would be de- 


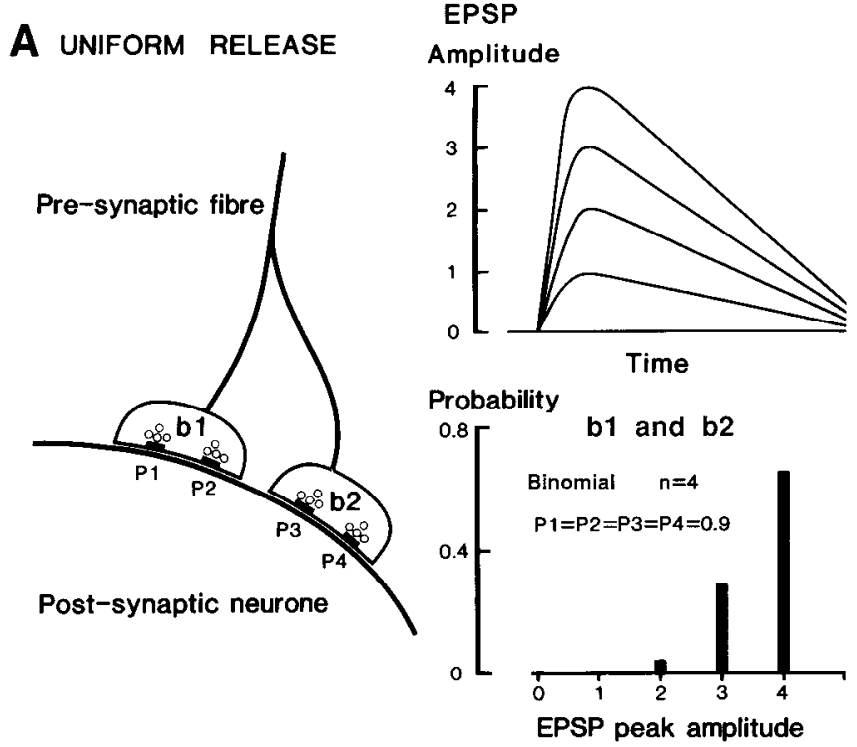

B PRE-SYNAPTIC INHIBITION

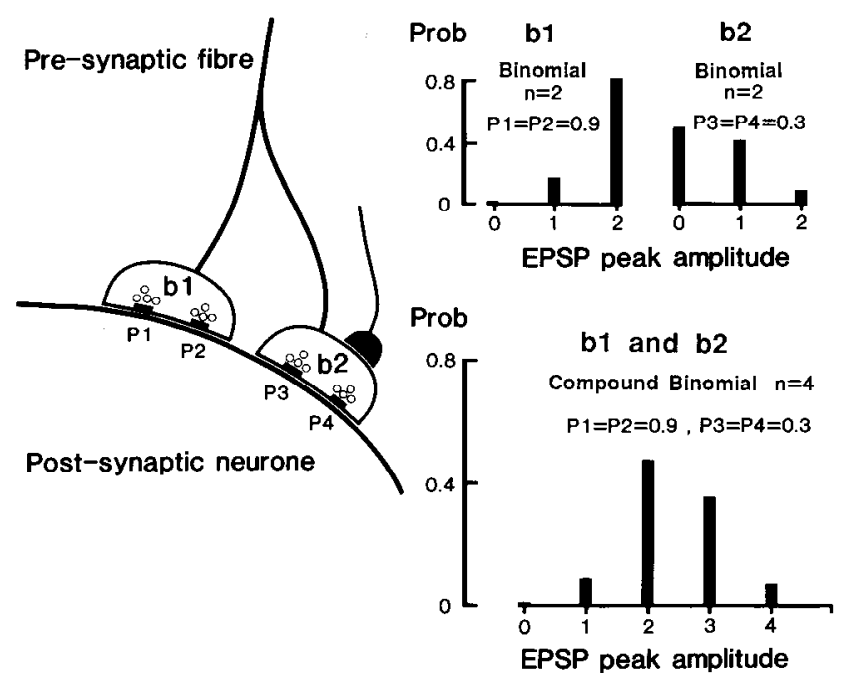

Figure 6. Summary of the possible effect of presynaptic contacts on synaptic transmission in the spinal cord. See text for explanation.

creased and, in this example, it has been arbitrarily reduced from 0.9 to 0.3 . The release probabilities from release sites in b1 have been assumed to remain at 0.9 . (The possibility exists for a polarization at $b 2$ to spread to $b 1$ and so influence the release probability at this bouton also, although to a lesser degree.) If it is now assumed that each bouton acts independently with simple binomial release probabilities (i.e., for b1, $n=2$ and $P 1=P 2=0.9$, and for $\mathrm{b} 2, n=2$ and $P 3=P 4=0.3$ ), then the overall fluctuation pattern could be described by a compound binomial model in which $n=4, P 1=P 2=0.9$ and $P 3=$ $P 4=0.3$, as shown in the lower right of Figure $6 B$.

Walmsley et al. (in press) have demonstrated that uniform binomial release statistics do not apply to the amplitude fluctuation pattern of single group I muscle afferent EPSPs recorded in DSCT neurons. Instead, the fluctuation pattern can be described if different release probabilities are assumed. The simple scheme presented in Figure $6 B$ represents only one explanation for such nonuniform release probabilities, based on the electronmicroscopic evidence presented in this study.

Continued investigation of the synaptic connection between primary afferent fibers and DSCT neurons will, we hope, allow us to formulate a general model of excitatory synaptic transmission in the mammalian CNS. The ultrastructural features of this connection provide a basis for our present investigations on the mechanisms underlying the probabilistic nature of synaptic transmission at this synapse.

\section{References}

Clements, J. D., I. D. Forsythe, and S. J. Redman (in press) Presynaptic inhibition of synaptic potentials evoked in cat spinal motoneurones by impulses in single group Ia axons. J. Physiol.

Curtis, D. R., and D. R. Lodge (1982) The depolarization of feline ventral horn group Ia spinal afferent terminations by GABA. Brain Res. 46: 215-233.

Curtis, D. R., B. D. Gynther, and R. Malik (in press) A pharmacological study of group Ia muscle afferent terminals and synaptic excitation in the intermediate nucleus and Clarke's column of the cat spinal cord. Exp. Brain Res.

Dudel, J., and S. W. Kuffler (1961) Pre-synaptic inhibition at the crayfish neuromuscular junction. J. Physiol. (Lond.) 155: 543-562.

Eccles, J. C., R. F. Schmidt, and W. D. Willis (1963) Pharmacological studics on presynaptic inhibition. J. Physiol. (Lond.) 168: 500-530.

Fyffe, R. E. W., and A. R. Light (1984) The ultrastructure of group Ia afferent fiber synapses in the lumbosacral spinal cord of the cat. Brain Res. 300: 201-209.

Houchin, J., D. J. Maxwell, R. E. W. Fyffe, and A. G. Brown (1983) Light and electron microscopy of dorsal spinocerebellar tract neurons in the cat: An intracellular horseradish peroxidase study. Q. J. Exp. Physiol. 68: 719-732.

Jack, J. J. B., S. J. Redman, and K. Wong (1981) The components of synaptic potentials evoked in cat spinal motoneurones by impulses in group Ia fibres. J. Physiol. (Lond.) 321: 65-96.

Jankowska, E., and Y. Padel (1984) On the origin of presynaptic depolarization of Group I muscle afferents in Clarke's column in the cat. Brain Res. 295: 195-201.

Jankowska, E., M. G. M. Jukes, and S. Lund (1965) On the presynaptic inhibition of transmission to the dorsal spinocerebellar tract. J. Physiol. (Lond.) 177: 19-20P.

Jankowska, E., D. McCrea, P. Rudomin, and E. Sykova (1981) Observations on neuronal pathways subserving primary afferent depolarization. J. Neurophysiol. 46: 506-516.

Redman, S. J., and B. Walmsley (1981) The synaptic basis of the monosynaptic stretch reflex. Trends Neurosci. 4: 248-250.

Rethelyi, M. (1970) Ultrastructural synaptology of Clarke's column. Exp. Brain Res. 11: 159-174.

Rudomin, P., I. Engberg, and I. Jimenez (1981) Mechanisms involved in the generation of presynaptic depolarization of group I afferent and rubro-spinal fibers in the cat spinal cord. J. Neurophysiol. 46: 532547.

Saito, K. (1974) The synaptology and cytology of the Clarke cell in the nucleus dorsalis of the cat: An electron microscopic study. J. Neurocytol. 3: 179-197.

Saito, K. (1979) Morphometrical synaptology of Clarke cells and of distal dendrites of the nucleus dorsalis: Electron microscopic study in the cat. Brain Res. 178: 233-249.

Szentagothai, J., and A. Albert (1955) The synaptology of Clarke's column. Acta. Morphol. Hung. 5: 43-51.

Tracey, D. J., and B. Walmsley (1984) Synaptic input from identified muscle afferents to neurones of the dorsal spinocerebellar tract in the cat. J. Physiol. (Lond.) 350: 599-614.

Walmsley, B., F. R. Edwards, and D. J. Tracey (in press) The probabilistic nature of synaptic transmission at a mammalian excitatory central synapse. J. Neurosci.

Walmsley, B., E. Wicniawa-Narkicwicz, and M. J. Nicol (1985) The ultrastructural basis for synaptic transmission between primary muscle afferents and neurones in Clarke's column of the cat. J. Neurosci. 5: 2095-2106. 